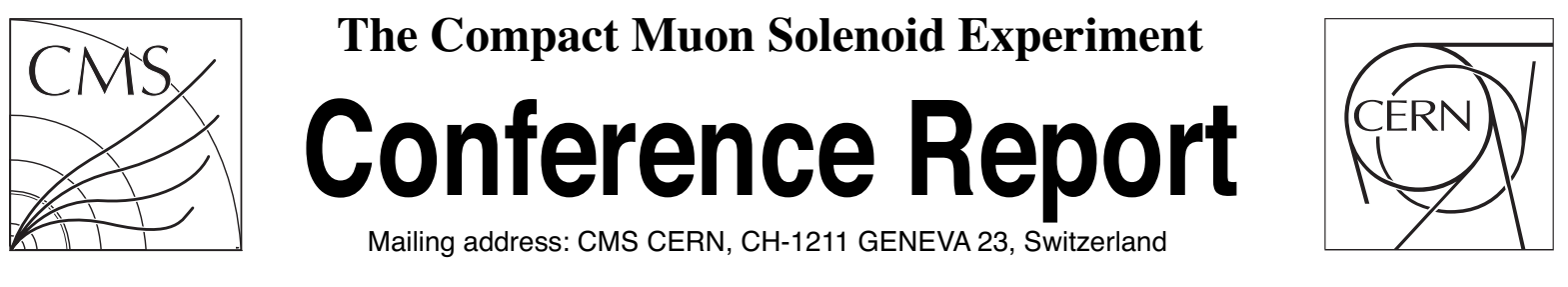

29 September 2014

\title{
The upgrade of the muon system of the CMS experiment
}

\author{
Marcello Abbrescia for the CMS Collaboration
}

\begin{abstract}
The CMS muon system is based on three types of gaseous detectors, RPC, CSC and DT. While operating very well in the present conditions, upgrades are foreseen for each of the subsystems, necessary to cope with the increased pile-up, coming along with higher rates and radiation, during the upcoming periods of data taking.Moreover, an important issue will be to make the system able to perform its delicate task of muon triggering and tracking also in the High Luminosity phase of LHC, foreseen to start after Long Shutdown 3 in 2023 and to last for about 10 years.Studies devoted to asses the system perfomance stability for the future will be presented. In addition, the stategy - which is being developed - to complement the existing system with new detectors, based on GEM or improved RPC technologies, will be shown.
\end{abstract}

Presented at PSD10 10th International Conference on Position Sensitive Detectors 


\title{
The upgrade of the Muon System of the CMS experiment
}

\author{
Marcello Abbrescia ${ }^{a}$ on behalf of the CMS collaboration \\ ${ }^{a}$ Dipartimento Interateneo di Fisica and Sezione INFN, \\ via Amendola 173, 70126 Bari, Italy \\ E-mail: marcello.abbresciadba.infn.it
}

\section{ABSTRACT:}

The CMS Muon System is based on three types of gaseous detectors, Resistive Plate Chambers, Cathods Strip Chambers and Drift Tubes.

While operating very well in the present conditions, upgrades are foreseen for each of the subsystems, necessary to cope with the increased pile-up, coming along with higher rates and radiation, during the upcoming periods of data taking.

Moreover, an important issue will be to make the system able to perform its delicate task of muon triggering and tracking also in the High Luminosity phase of LHC, foreseen to start after Long Shutdown 3 in 2023 and to last for about 10 years.

Studies devoted to asses the system perfomance stability for the future will be presented. In addition, the strategy - which is being developed - to complement the existing system with new detectors, based on Gas Electron Multipliers or improved RPC technologies, will be shown.

KeYwords: CMS experiment; Muon System; Resistive Plate Chamber, Cathode Strip Chambers, Drift Tubes, Gas Electron Multipliers.

\footnotetext{
${ }^{*}$ Corresponding author.
} 


\section{Contents}

1. Introduction 囵

2. Upgrade of the existing detectors

3. GEM detectors for the GE1/1, GE2/1 and ME-0 stations

4. Improved RPCs for the RE3/1 and RE4/1 stations 廿

5. Conclusions 9

\section{Introduction}

The Muon System of the CMS experiment at the Large Hadron Collider (LHC) was designed to be robust, efficient and redundant, and to reliably provide muon identification, measurement of the muons transverse momentum, and correct bunch crossing assignment. It consists of three types of muon detectors: Drift Tubes (DTs), Cathode Strip Chambers (CSCs), and Resistive Plate Chambers (RPCs), as shown in Figure 1] and described in details in [1]. The system provided an excellent performance throughout the first period of data taking (so-called Run1), significantly

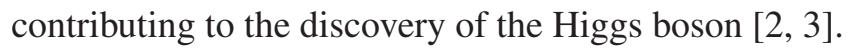

It was originally designed, like the whole CMS experiment, for ten years of operation at $1 \times$ $10^{34} \mathrm{~cm}^{-1} \mathrm{~s}^{-1}$ corresponding to a maximum of $500 \mathrm{fb}^{-1}$ integrated luminosity. However, during the Long Shutdowns 2 and 3 (LS2 and LS3), respectively foreseen in 2018 and around 2023, the LHC injector chain will be significantly improved, and new low $\beta$ and crab cavities will be installed. After LS3, the instantaneous luminosity will be around $5 \times 10^{34} \mathrm{~cm}^{-1} \mathrm{~s}^{-1}$ with a corresponding integrated luminosity of about $300 \mathrm{fb}^{-1} /$ year. This period, expected to last for further ten years at least, is referred to as the High Luminosity phase of LHC (HL-LHC)

Operating in these new harsher conditions will represent a major challenge for CMS, due, for instance to the higher radiation background and the increased Pile-Up (PU). Therefore the CMS collaboration is putting a considerable effort to study the issues associated with the HL-LHC operation, and the optimal strategy to cope with them. For instance, the tracking system and the endcap calorimeters will be upgraded, due to large radiation dose integrated in these regions; also the trigger system will have to be upgraded. These upgrades are described in details in 四].

For what concerns the Muon System, three types of upgrades are proposed for HL-LHC:

1. upgrades of existing muon detectors and associated electronics that should ensure their longevity and good performance also during HL-LHC; 


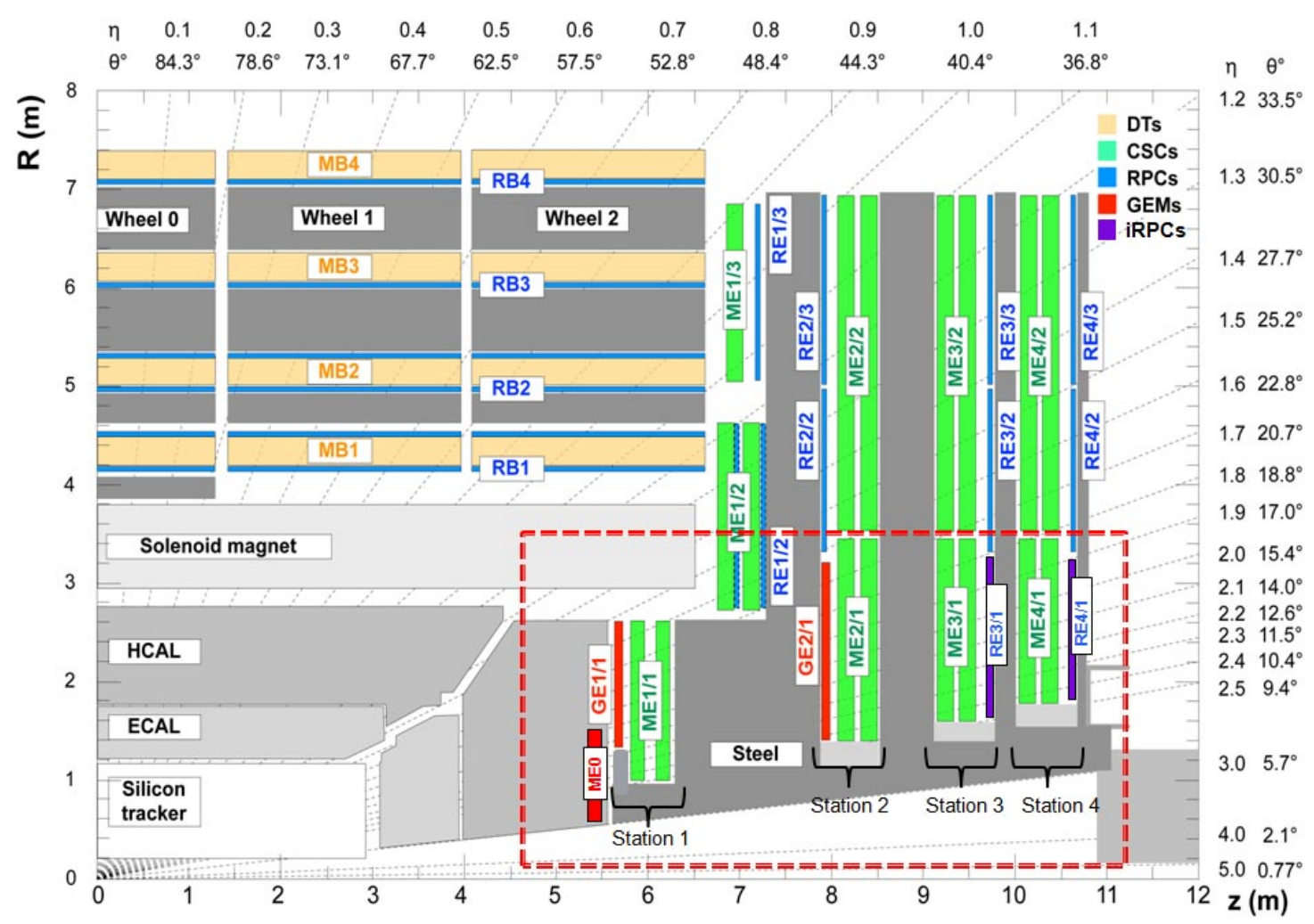

Figure 1. A quadrant of the muon system, showing DT chambers (yellow), RPC (light blue), and CSC (green). The locations of new forward muon detectors for HL-LHC phase are contained within the dashed box and indicated in red for GEM stations (ME0, GE1/1, and GE2/1) and dark blue for improved RPC stations (RE3/1 and RE4/1).

2. additional muon detectors in the forward region in the pseudorapidity range $1.6<|\eta|<$ 2.4 to increase redundancy and enhance the trigger and reconstruction capabilities (stations GE1/1 and GE2/1, stations RE3/1 and RE4/1, indicated in Figure (1);

3. extension of muon coverage up to $|\eta|=3$ or more behind the new endcap calorimeter (station ME-0, also indicated in Figure (1).

This paper briefly reviews the interventions foreseen, with particular reference to the technological improvements from the detector point of view, which will be put in place.

\section{Upgrade of the existing detectors}

In order to assess their longevity and performance stability up to an integrated luminosity of 3000 $\mathrm{fb}^{-1}$, both DTs, CSCs and RPCs are planning extensive campaigns of irradiation. These will be carried out at a new facility, the $\mathrm{GIF}^{++}$at CERN [5], which will come into operation at the beginning of 2015. At $\mathrm{GIF}^{++}$, detectors and material samples can be irradiated by $662 \mathrm{keV}$ photons emitted by an intense $16.7 \mathrm{TBq}{ }^{137} \mathrm{Cs}$ source. A high-momentum particle muon beam extracted 

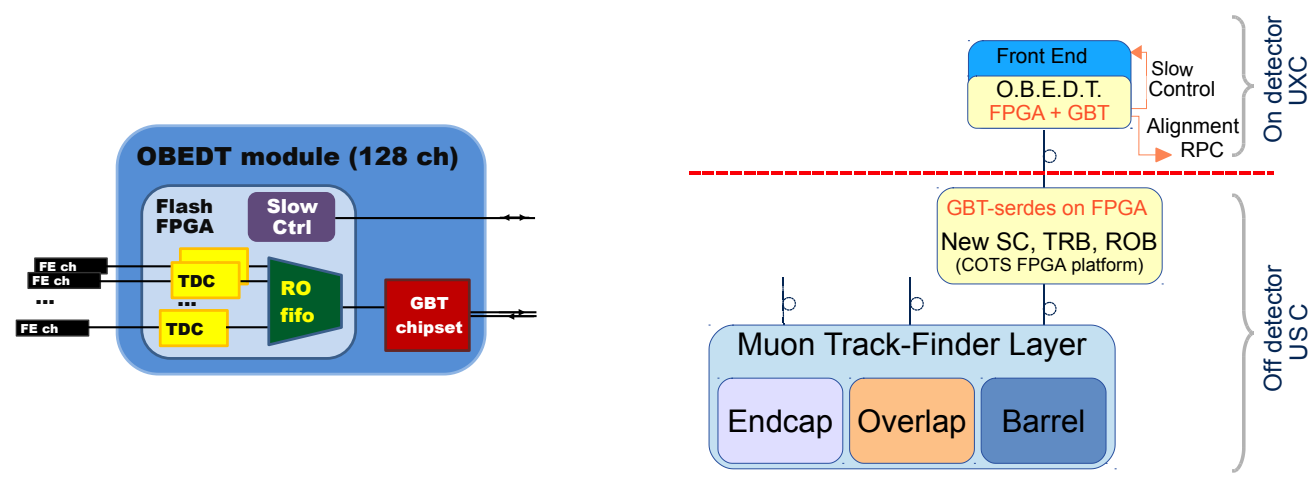

Figure 2. Block diagrams of proposed DT electronics. Left: scheme of on-board electronics (OBEDT). Right: overall view of the on-detector and off-detector components of the proposed architecture.

from the SPS, will be used to study the detectors performance on ionizing particles while these are irradiated with photons, simulating the high background conditions which will be present at CMS during HL-LHC.

In addition to the above mentioned aging studies, each sub-detector has foreseen specific upgrades.

DTs upgrade plans are mainly focused on electronics. Each DT chamber houses the L1 Trigger and Readout electronics in an aluminum profile, called a Minicrate [6], which hosts the time digitization logic and the logic for the L1 trigger primitive generation. During HL-LHC the survival of this system is not guaranteed, and will not be able to cope with increasing the Level 1 trigger acceptance rate beyond $300 \mathrm{kHz}$, as it us foreseen. Consequently, the substitution of this electronics is planned for LS3, moving the trigger logic to the service cavern.

Moreover, this replacement will allow the implementation of full time (and therefore space) resolution and to provide complete chamber information in the DT trigger system, with improved performance in terms of rate reduction and better matching with the tracker at the Level 1 trigger.

The functionalities that will remain close to the DT chamber are: time digitization of the chamber Front-End Boards (FEBs) analog signals, control of the FEBs, test pulse generation, monitoring of DT chamber sensors (temperature, pressure, etc.), and slow control services for the RPC and Alignment systems. These will be implemented in a single type of board (Figure —, left).

Once digitized, hit times will be sent to the underground control room (USC) through an optical connection. In the proposed upgrade, the trigger primitive generation and the readout event matching will be performed in the underground control room on generic hardware, based on the latest commercial FPGAs. A diagram of this system is shown in the right side of Figure \&.

CSC upgrade plans are also focused on electronics, in particular foreseeing the replacement of cathode front-end boards (CFEB) on inner chambers in order to handle the increased L1 trigger latency and rate. The CFEB use analog charge storage within custom Switched Capacitor Array (SCA) chips (ASICs) that sample at 50 ns intervals and contain a depth of 96 cells. These boards are used on all CSCs except ME1/1 chambers which, in 2013-2014, have undergone an upgrade of the front- and back-end electronics to improve rate capability and performance at higher luminosity. With the increased L1 trigger latency (up to $12.5 \mathrm{~ms}$ ) and rate (up to $750 \mathrm{kHz}$ ) that is planned for 
HL-LHC CMS operation, the 108 inner CSC chambers (ME2/1, ME3/1, and ME4/1) will often fill the SCA cells; consequently no additional data can be stored, causing readout inefficiency.

Therefore, the CFEBs will be replaced by boards similar to the "digital" DCFEBs that have already been installed on the ME1/1 chambers. These boards flash-digitize the data continuously and store them in large digital buffers, resulting in zero deadtime and the capability to handle latencies well beyond the current specification. They also send their output data on $3.2 \mathrm{~Gb} / \mathrm{s}$ optical links, as compared to $1 \mathrm{~Gb} / \mathrm{s}$ achievable on the copper output cables of the CFEBs; the higher output data capability of the DCFEBs is important to handle the HL-LHC and CMS L1 trigger conditions.

Some additional elements of the CSC data acquisition system, namely the Data Acquisition Motherboards (DMBs) and Detector-Dependant Units (DDUs) that connect to the DCFEBs will will be replaced as well in order to handle the high data throughput using the fast optical links.

Among other activities, RPCs will mainly focus on the investigation and qualification of a new gas mixture needed to fulfill new environmental regulations. The CMS RPC gas mixture is a three-component mixture of $95.2 \% \mathrm{C}_{2} \mathrm{H}_{2} \mathrm{~F}_{4}$ (Freon R-134a, tetrafluoroethane), $4.5 \% \mathrm{C}_{4} \mathrm{H}_{10}$ (isobutane) and $0.3 \% \mathrm{SF}_{6}$ (sulfur hexafluoride), humidified at about $40 \%$. Recently, because of its high Global Warming Potential (GWP) of 1430 (with respect to $\mathrm{CO}_{2}$ ), the use of R-134a is increasingly restricted by international regulations following the 1997 Kyoto Protocol.

Possible alternatives are already under study such as the R-152a, with a GWP of 120, and the most promising ones HFO-1234ze and HFO-1234yf (tetrafluoropropane) which are non-ozone depleting and with a very low GWP (6 and 4, respectively). R\&D tests with new gas mixtures have been recently started in some laboratories of the CMS collaboration, aimed at exploring these, and other, eco-gas candidates. Since the gas mixture is the core of a gaseous detector, replacing it is a non-trivial issue, and needs careful study to optimize again the detector working conditions with the new gas mixture. Moreover, in high-radiation environments like those expected at the HL-LHC, several new chemically-reactive impurities could be created inside the RPC, for instance HF, which are potentially harmful to the detector materials and ultimately may degrade the detector performance. Therefore accurate measurements of production rate and design of the appropriate filters to remove them will be put in place.

\section{GEM detectors for the GE1/1, GE2/1 and ME-0 stations}

GEMs are gaseous detectors, characterized by a spatial resolution of order $100 \mathrm{~mm}$, time resolution of a few ns, and detection efficiency more than $90 \%$ even for rate exceeding few $\mathrm{MHz} / \mathrm{cm}^{2}$. Despite being invented just in 1997, a long term experience on the field has been accumulated in LHCb, COMPASS and TOTEM; in addition they can be operated with a non-flammable gas mixture $\left(\mathrm{Ar} / \mathrm{CO}_{2} / \mathrm{CF}_{4}\right)$, which is an added value in the underground caverns of the LHC experiments.

For HL-LHC, GEM detectors are proposed to instrument two forward stations, called GE1/1 and GE2/1 and indicated in Figure [1. The GE1/1 and GE2/1 detectors are "superchambers", each made of a double layer of trapezoidal triple-GEM chambers. They cover slightly more than $10^{\circ}$ in GE1/1 and $20^{\circ}$ in GE2/1, overlapping in $\phi$ just like the corresponding CSC chambers in stations ME1/1 and ME2/1, complementing them and providing redundancy, enhanced triggering and reconstruction capabilities. For both endcaps, 72 superchambers will be needed for GE1/1 and 36 for 

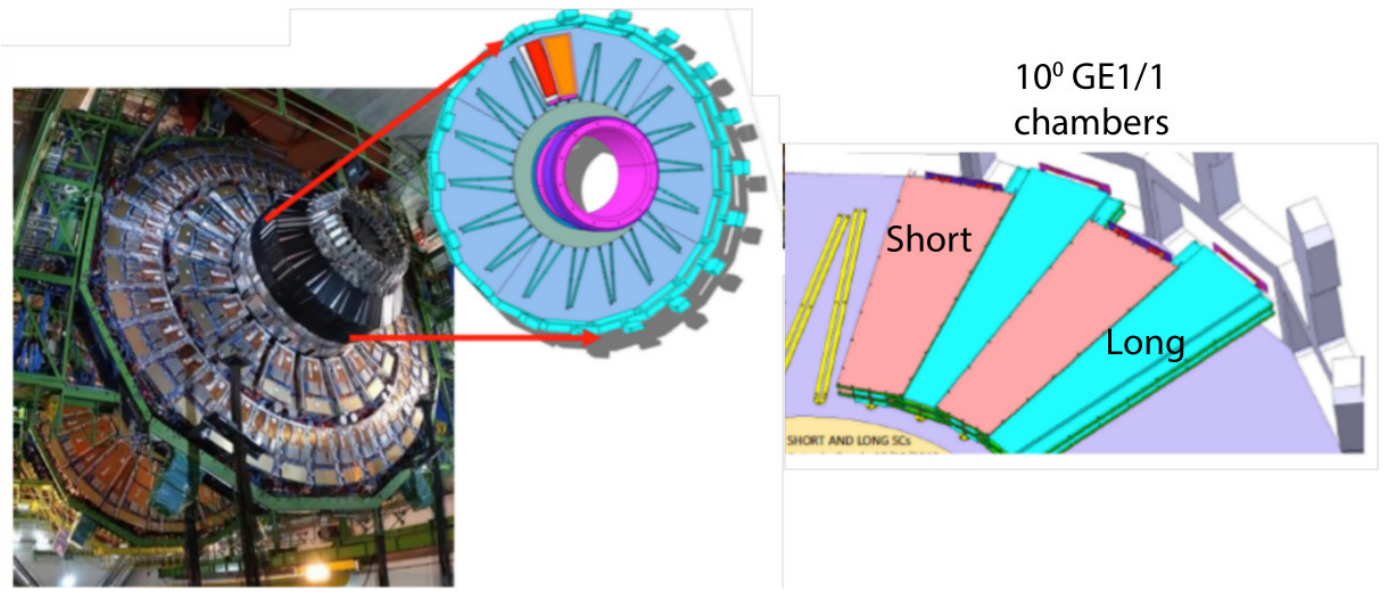

Figure 3. Left: location of the GE1/1 detectors on the YE1 nose. Right: detailed view of the overlapping of GE1/1 superchambers.
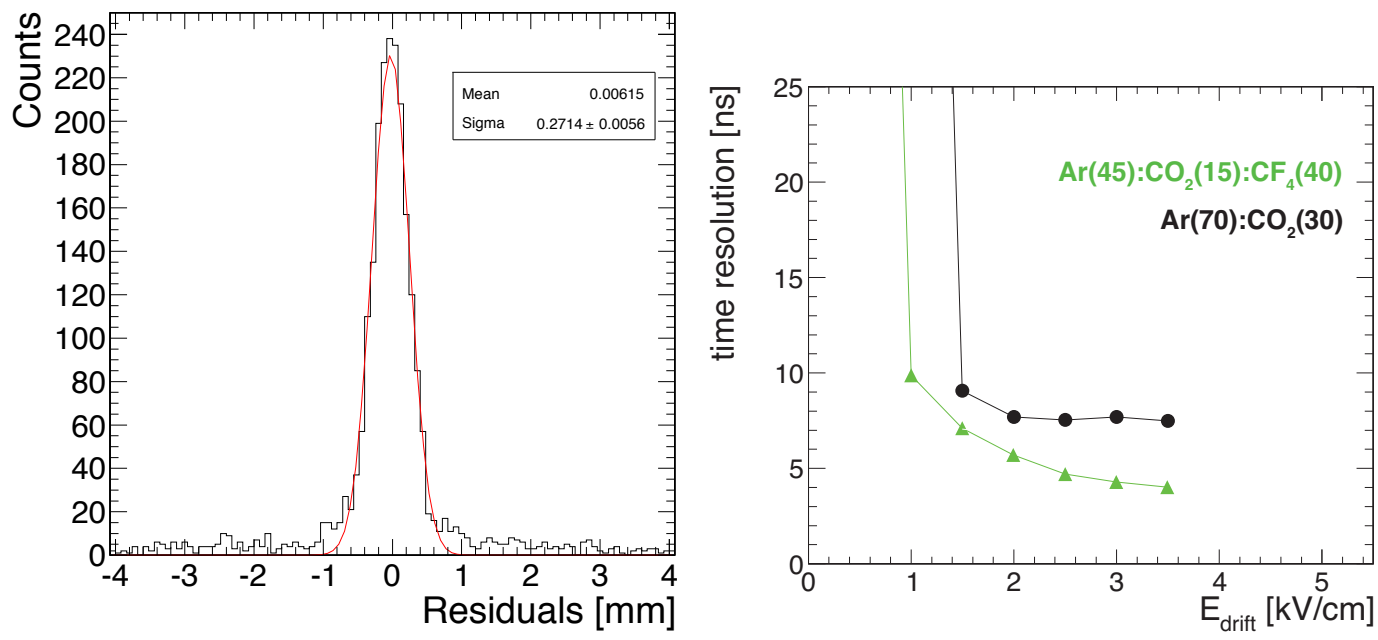

Figure 4. Triple-GEM resolutions obtained in a GE1/1 prototype using binary readout. Left: residual distribution with a fitted $\sigma=270 \mu \mathrm{m}$. (right) Timing resolution as a function of drift field for two representative gas mixtures, $\mathrm{Ar} / \mathrm{CO}_{2}$ with (5 ns) and without (7-8 ns) the addition of $\mathrm{CF}_{4}$.

GE2/1. The location of these chambers on the "nose" of the first endcap disk of CMS is shown in Figure 3 (left), and a closeup of the GE1/1 short and long detectors within the nose is presented in Figure 3 (right).

In order to make GEMs detectors suitable for HL-LHC, in the context of the CMS GEM R\&D effort (CMS RD10.02), many technological detector break-troughs have been achieved. For instance production of large-area GEMs with the single-mask technique has been demonstrated [Z]. Moreover, a novel spacerless and stretching technique has also been developed that allows production of large-area triple-GEM detectors without any gluing: the three GEM foils are mounted into a single stack where they are kept together with a segmented frame, also providing the inter-GEM spacing. This construction technique allows assembly of one full-scale GE1/1 chamber in about 


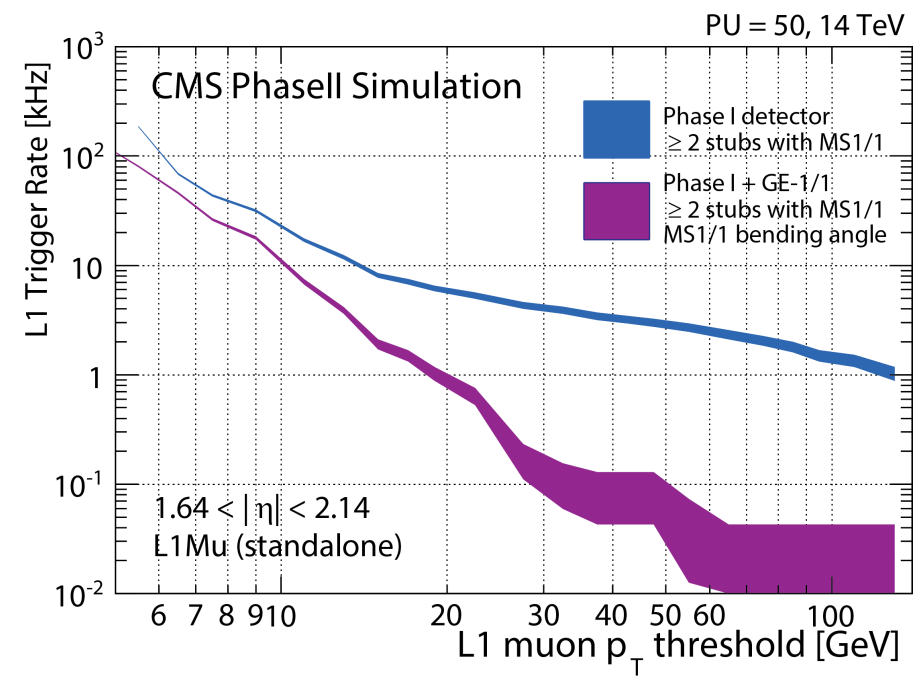

Figure 5. (left):L1 muon trigger rate at a luminosity of $2 \times 10^{34} \mathrm{~cm}^{-2} \mathrm{~s}^{-1}$ as a function of $p_{t}$ threshold. For the present system, 2 or more stubs, one of which is in the ME1/1 station are required. With the addition of GE1/1, the bending angle between the two stations can be used and the trigger rate is greatly reduced.

two hours, compared to one week for a detector assembled with the conventional gluing technique. Furthermore using mechanically stretched and spacerless technology allows easy reopening of a chamber if necessary.

Several prototypes have been assembled and tested in the lab with x-rays and with test beams [8] during the last four years. Just as an example, the performance of a standard triple-GEM detector built with the single-mask technique, using gas mixture of $\mathrm{Ar} / \mathrm{CO}_{2} / \mathrm{CF}_{4}(45 / 15 / 40 \%)$, and running at a gas gain of $10^{4}$ (achieved at a total applied voltage of $3.8 \mathrm{kV}$ between the drift cathode and the readout PCB), is shown in Figure $\$$. On the left a spacial resolution of $270 \mu \mathrm{m}$ with binary readout in a part of a GE1/1 prototype section that has a strip pitch of $0.9 \mathrm{~mm}$ is shown, and on the right time resolution of 5 and $8 \mathrm{~ns}$ at plateau for gas mixtures with and without $\mathrm{CF}_{4}$, respectively, is demonstrated.

In addition to GE1/1 and GE2/1, the ME0 station is proposed to be instrumented with GEMs and installed in a space of $\sim 30 \mathrm{~cm}$ freed behind the new endcap calorimeters. The coverage is foreseen to extend up to $\eta=3$, or more, depending on the boundary defined by these calorimeters. A six-layer device is foreseen, to allow proper rejection of neutron background, very intense in that region. The design of ME0 incorporates neutron and photon shielding using, for example, borated polyethylene and lead. The detector will be made of units of $20^{\circ}$ wedges with inner and outer radii of 300 and $1495 \mathrm{~mm}$.

Many are the reasons while upgrade with GEMs are needed for HL-LHC or even earlier, and they are described in details in [田]. Just an example, the blue curve in Figure 5 shows the L1 muon trigger rate at a luminosity of $2 \times 10^{34} \mathrm{~cm}^{-2} \mathrm{~s}^{-1}$ as a function of $p_{t}$ threshold, in the case the present system configuration is left unchanged. An efficient trigger (with a plateau efficiency of 94\%) would require a too-high-bandwidth allocation of $>20 \mathrm{kHz}$ out of the total L1 trigger acceptance rate of $100 \mathrm{kHz}$, which is not acceptable. 

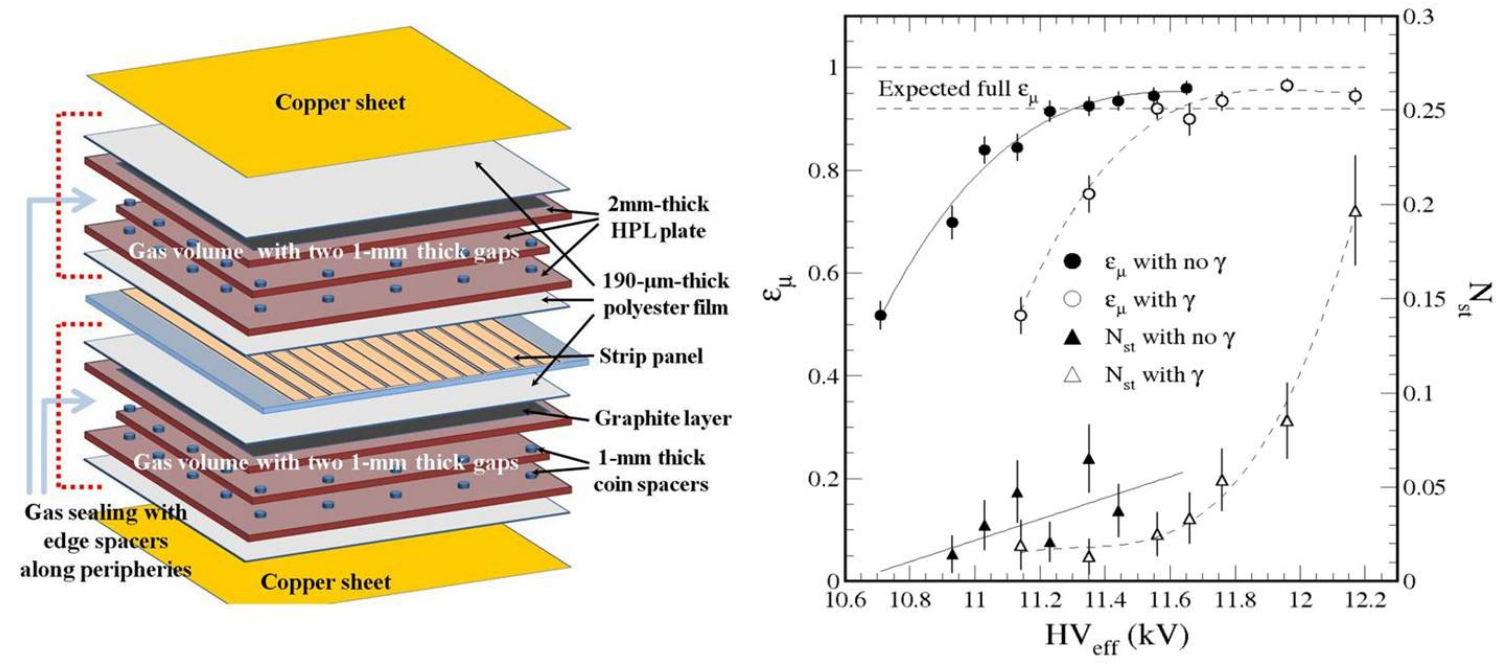

Figure 6. Left: view of a multigap HPL RPC prototype. Right: efficiency plateau (circles) and streamer probability (triangles) vs. operating voltage with (open symbols) and without (solid symbols) a $3 \mathrm{kHz} / \mathrm{cm}^{2}$ $\gamma$ irradiation.

On the contrary, installation of the GE1/1 station will address most of the causes of the trigger performance degradation. In addition to implementing the redundancy necessary for efficient selection of muons in the most critical region, it will allow improved measurement of the muon bending angle in the first station, using the lever arm formed by the GEMs and the CSCs. This will reduce the tails in the transverse momentum resolution, and therefore the trigger rate as illustrated in Figure 5. With this upgrade, CMS will be able to maintain a single-muon trigger threshold of $p_{t} \sim 15 \mathrm{GeV}$, ensuring full efficiency for offline reconstruction of muons with $p_{t}>20 \mathrm{GeV}$.

\section{Improved RPCs for the RE3/1 and RE4/1 stations}

Improved versions of the present RPCs, usually indicated as iRPCs, capable of handling the particle rates foreseen during HL-LHC, are considered to instrument the RE3/1 and RE4/1 stations of the CMS endcaps (see again Figure 11). The new chambers would complement the existing ME3/1 and ME4/1 stations, currently instrumented with CSCs only, providing improvements to the L1 muon trgger and redundancy in a most critical region.

First results provided by FLUKA simulations, and the comparison with data from the CSCs, provide an estimate of the rate around $700 \mathrm{~Hz} / \mathrm{cm}^{2}$ in the hottest points of RE3/1 and RE4/1. Taking into account an additional safety factor, a rate capability of around $2 \mathrm{kHz} / \mathrm{cm}^{2}$ should be required for the new chambers, while standard CMS double-gap RPCs have been tested to reach around $1 \mathrm{kHz} / \mathrm{cm}^{2}$ (see, for instance, [9]). Therefore an improvement in the rate capability of these detectors is needed.

The rate capability of RPCs (see [11]) can be improved in various ways:

1. Reducing the electrode resistivity $\rho$, which has the direct effect of reducing the recovery time needed for the electrodes to be charged up again after a discharge in the gas gap; this 


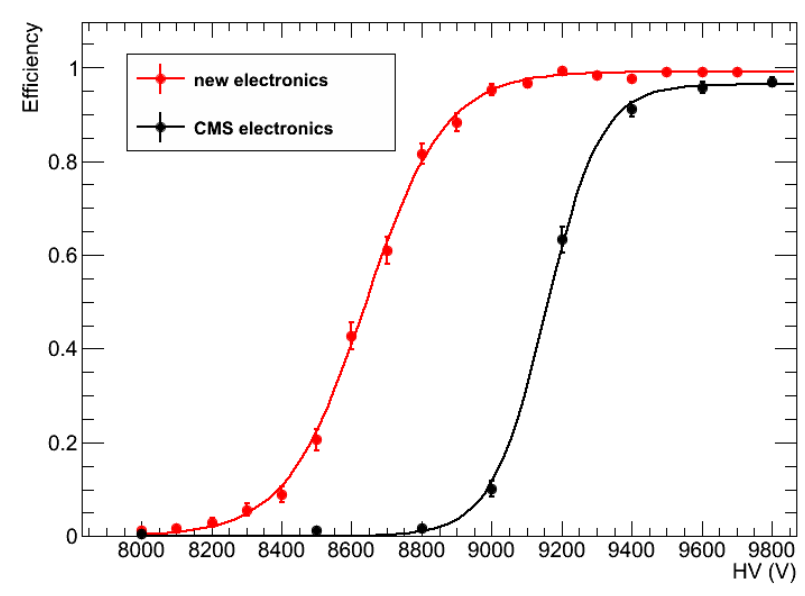

Figure 7. Efficiency plateaus for the cases of the standard CMS RPC (black points) and new ATLAS prototype (red points) electronics. The shift in the beginning of the plateau toward the left indicates that in this case a lower charge is crossing the RPC gap. This can help to improve the rate capability of the RPC.

increases the rate capability proportionally.

2. Reducing the average charge generated in the avalanches, and transferring part of the signal amplification from the gas to the front-end electronics. This also reduces aging processes.

3. Changing the detector configuration, which includes many possible options, like changing the electrode thickness or their number (multigap). For a given induced signal (i.e. detector efficiency), this increase the ratio of the induced signal to the moving charge in the gap, with beneficial effects similar to those previously described.

The final technique adopted in CMS may combine the benefits of the different approaches.

The resistivity of the high-pressure laminates (HPL) made with Bakelite resin used in the present CMS RPC detectors is $1-5 \times 10^{10} \Omega \mathrm{cm}$. Recent production had resistivities near the low end of this range. By adjusting the proportions of phenol-impregnated paper and melamine resin in the production process, a range of $0.5-1 \times 10^{10} \Omega \mathrm{cm}$ might be achievable. Prototype detectors made using this lower-resistivity HPL will soon be produced and tested.

One version of higher-amplification electronics has been developed in the framework of the muon system upgrade of the ATLAS experiment [12]. Tests comparing the performance of CMS chambers equipped with the standard CMS electronics and an early prototype of this new electronics have been performed with cosmic rays; a shift of about $460 \mathrm{~V}$ in the efficiency curves is obtained (see Figure 7). This is a strong hint at an improved rate capability, that will be tested in realistic conditions at the upcoming GIF++ facility.

The changes to the RPC detector configuration that are considered to enhance the rate capability are thinner electrodes (from $2 \mathrm{~mm}$ at present to 1.5 or even $1 \mathrm{~mm}$ ) with double-gap HPL, and multi-gap configurations using HPL and glass with thin gaps and electrodes. Thinner electrodes increase the signal induced on the readout strips and, subsequently, improve the rate capability. 


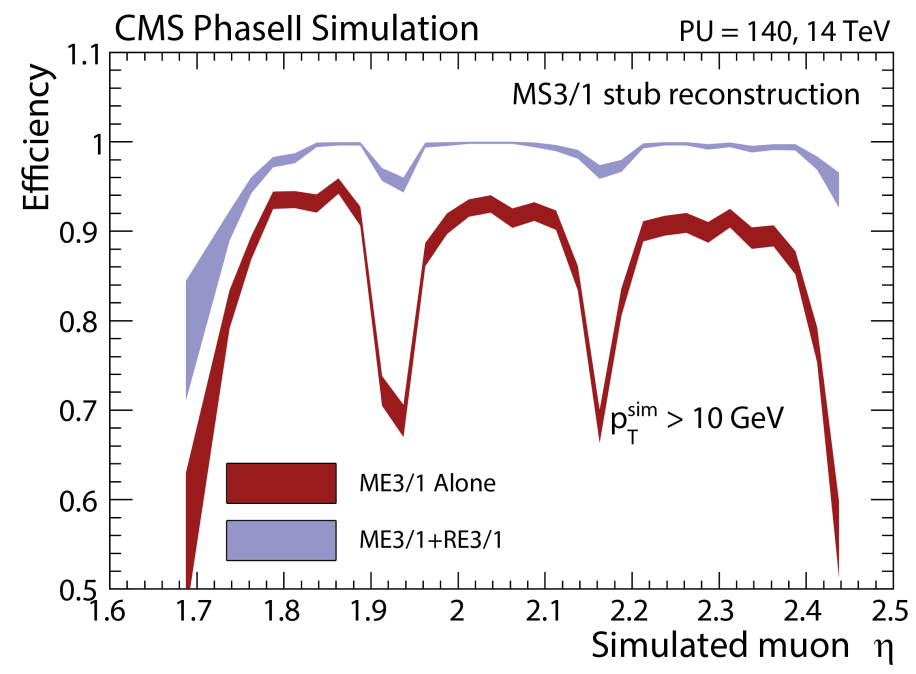

Figure 8. The local-trigger primitive ("stub") reconstruction efficiency in station 2 as a function of eta for the present detector (red curve) and with the addition of RE4/1 (blue curve).

Multi-gaps divide the charge necessary to induce a signal higher than the electronics threshold in more than one gap, and enhance the rate capability as well.

A schema of one multi-gap HPL detector tested in the the framework of the CMS/RPC R\&D is shown in Figure 6 (left). The standard double-gap configuration used in CMS has been modified by adding an additional gap on both sides of the readout strips. In this particular case, thickness of each of the four gaps is $1 \mathrm{~mm}$, the material used for the eletrodes is identical to the one already used for CMS/RPC chambers (same resistivity), and the readout electronics as well. Efficiency for cosmic rays muon vs. operating voltage in the two cases of no irradiation and irradiation with a 150 $\mathrm{mCi}{ }^{137} \mathrm{Cs} \gamma$-ray source providing a hit rate around $3 \mathrm{kHz} / \mathrm{cm}^{2}$ on the whole chamber is shown in Figure 6 (right). The chamber reaches full efficiency and has a plateau several hundreds volts wide in both cases, with a reduced $(<10 \%)$ streamer probability. Several other results are described in details in [13].

Again, reasons that drive to install these new stations for the HL-LHC are described in details in [四]. Just as an example, the plots in Fig. 8 shows that at $140 \mathrm{PU}$ (as foreseen during HL-LHC), even for a perfectly working existing system (red curves), the typical reconstruction efficiency drops below $90 \%$ with significant dips due to the high-voltage spacers inside the CSCs. The same figure shows that the installation of station RE3/1 (the RE4/1 case is very similar to RE3/1) restores the local-reconstruction (stub) efficiency.

\section{Conclusions}

During Run 1, the CMS Muon systems has been operating extremely well, delivering good data for physics. After 3 years of LHC running with increasing instantaneous luminosity and 6 years from the end of construction, the detector performance is within specifications both for triggering and as a reconstruction system. 
Neverthless, keeping the system performance at the same level also during HL-LHC will be a considerable challenge. Efforts to consolidate the present system in order to assure its longevity also during HL-LHC have already started.

In addition, new detector technologies are proposed to be installed in still empty locations. Triple-GEMs are proposed to instrument the $1.5<|\eta|<2.4$ region of the CMS forward muon system with high spatial resolution detectors that complement the existing Cathode Strip Chambers detectors, providing redundancy, enhanced triggering and reconstruction capabilities; an additional station, called ME-0 is proposed behind the calorimeters, to increase pseudorapidity coverage.

In RE3/1 and RE4/1, an improved version of RPCs capable to handle the high particle rates foreseen during the HL-LHC phase will complement the existing ME3/1 and ME4/1 stations, currently instrumented with CSC only, providing robustness to the L1 muon trigger where redundancy is missing, and ensuring constant performance even during HL-LHC.

\section{References}

[1] CMS collaboration, CMS The Muon Project Technical Design Report, CERN-LHCC-1008-032, CMS-TDR-3

[2] CMS collaboration, The performance of the CMS muon detector in proton-proton collisions at sqrt(s)=7 TeV at the LHC, JINST 7 (2013) P100002.

[3] CMS Collaboration, Performance of CMS muon reconstruction in pp collision events at sqrt $(s)=7$ TeV, JINST 7 (2012) P10002.

[4] CMS Collaboration, Technical Proposal for the Phase-II upgrade of the Compact Muon Solenoid, in print.

[5] M. Capeans et al., Proposal to the CERN SPSC: A GIF++ Gamma Irradiation Facility at the SPS H4 Beam Line, SPSC-2009-029 / SPSC-P-339 (2009).

[6] CMS collaboration, CMS Technical Design Report for the Trigger and Data Acquisition project, Volume I, CERN-LHCC-2000-038, CMS-TDR-6.1 (2000) page.

[7] D. Abbaneo et al., Characterization of GEM Detectors for Application in the CMS Muon Detection System, Nucl. Sci. Symp. Conf. Rec. 1416-1422 (2010).

[8] D. Abbaneo et al., Test Beam Results of the GE1/1 Prototype for CMS High- $\eta$ Muon System Future Upgrade, IEEE Nucl.Sci.Symp.Conf.Rec. 1806-1810 (2011).

[9] M. Abbrescia et al., Study of long-term performance of CMS RPC under irradiation at the CERN GIF, Nucl. Instrum. Meth. A533 (2004) 102.

[10] H.C. Kim et al., Quantitative aging study with intense irradiation tests for the CMS forward RPCs, Nucl. Instrum. Meth. A602 (2009) 771.

[11] M. Abbrescia, The dynamic behaviour of Resistive Plate Chambers, Nucl. Instrum. Meth. $\mathbf{A 4 5 3}$ (2004) 7.

[12] R. Cardarelli et al., Performance of RPCs and diamond detectors using a new very fast low noise preamplifier, JINST 8 P01003 (2013).

[13] K. S Lee at al., Rate capability study for a four-gap phenolic RPC with a CS-137 source, in print on JINST contribution to RPC2014 (2014). 